\title{
Entgegnung an Herrn Jakob Juratzka in Wien.
}

\section{Von Ludwig v. Farkas-Vukotinovic.}

* Es war mir durchaus nicht unlieb, Hrn. J u r a $t \mathrm{z} k$ a's freundliche Bemerkungen über die von mir aufgestellte Schlosseria zu lesen; durch einen Austausch der Ansichten kann die Sache nur gewinnen und in's Reine gebracht werden. Der firagliche Gegenstand kann von zwei Gesichtspunkten betrachtet werden. Nehmen wir den streng wissenschaftlichen Gesichtspunkt an, der nur der naturhistorische sein kann, so bekommt die Sache ein ganz anderes Licht; ich kann da nichts anderes sagen, als dass die naturhistorische Achnlichkeit der Specierum ein Genus bilde, und dass dann sehr viele Genera (nämlich jetzt in der Botanik bestehende) bei den Umbellisten zusammengezogen werden, weil sie wirklich eine solche Aehnlichkeit besitzen, dass die allenthalben gebrauchten Unterscheidungs-Merkmale unbeachtet bleiben müssen; da dürfen wir kein Coriandrum yon Bifora; keinen Anthriscus von Torilis und Chaerophyllum, kein Peucedanum von Pteroselinum, kein Chironium von Pastinaca, kein Laserpitium von Siler u. s. w. trennen; demn es ist in der Wirklichkeit kein Grund dazu vorhanden; diess ist meine Ueberzeugung, und es wäre $z u$ wünschen, dass wir alle diesen Grundsatz befolgen und ihn überall mit gleicher Consequenz durchführen würden.

Bei einer Beobachtung aber des Verfahrens, welches sich die Hrn. Botaniker gewöhnlich gefallen lassen, ist es nicht schwer zu bemerken, dass sie in manchen Fällen sehr liberalin manchen wieder sehr difficil sind. Es dürfte vielleicht eine kleine Inconsequenz im Verstecke liegen. Diesen Gedanken festhaltend, spielte ich darauf an, indem ich in Nr. 44 v. J. p. 350 sagte; , und da sie (Schlosseria) bei der gegenwärtigen Eintheilung der Umbellisten unter kein Genus regelrecht untergebracht werden kann", würde also bei der Gruppirung der Specierum das naturhistorische Princip angewendet, so fiele die beabsichtigte Aufstellung der neuen Gattung von sich selbst weg.

Der zweite Gesichtspunkt ist der allgemein beliebtere, den ich mir den derWillkürlichkeit zu nennen erlaube; dafür sprechen Hunderte von Namen, die voǹ den Einen gegeben, von denAndern genommen wurden. Wegen eines kleinenUnterschiedes in achenio oder in pappo, wegen einer Abweichung in calyce, silicula oder utriculo, vallecula u.s.w. sehen wir ein neues Genus, einen neuen Namen entstehen, den die Einen annehmen, die Andern ignoriren. Diess ist gewiss keine Tugend, kein Vortheil, es ist eine Willkürlichkeit, es' ist ein Fehler, den vielleicht um der Mode zu huldigen auch ich begangen haben mag! Hrin. Ju ratzka's freundliche Bemerkungen mögen eben nicht ganz unrichtig sein. Ganz ohne Grund ist aber meine Ansicht auch nicht. Die fragliche Pflanze hat Anlass zu mehrfachen Forschungen gegeben; sie wurde bald zu Pastinaca, bald zu Peucedanum, bald zu Re i$\mathrm{c}$ h en bach's Pteroselinum gezählt, endlich auch als eigenes Genus Palimbia aufgestellt. Das wären also schon vier Genera; sicherlich 
fand man jedesmal dazu einen Grund, wenn auch nur einen kleinlichen; und ein kleinlicher genügt ja, wie es die Praxis hinlänglich beweiset.

Hr.v. Vis i a ni sagt zwar, wie auch Hr. Ju ratzk a anzuführen beliebt: Fructibus acutioribus planta haec recedit quidquam a specie, sed praeter hanc notam, quae parvi facienda, in reliquis convenit. Aber weiter sagt Hr. v. Vi i i a $n$ i noch : Hinc a Peucedano sola exiguitate dentium calycinorum diversa. Wir haben hier also dennoch zwei Unterschiede: Fructus acutiores und dentes calycinos, die, wenn auch klein, dennoch genug deutlich sind; endlich sind die Wurzelblätter in Anbetracht zu nehmen, die von Peucedanum chabraei wohl auch in etwas verschieden sein dürften.

H o st nannte diese Pflanze Seseli pimpinelloides! wahrscheinlich wegen den meistens breiten Wurzelblättern, und ich glaubte sie Schlosseria heterophylla nennen zu dürfen. Es ist jedenfalls etwas an der Pflanze, was zu diesen schwankenden Ansichien Grund gab, das lässt sich nicht wegläugnen, so wie ich auch das hervorheben zu können glaube, dass man zu weit herabging, indem man von der Aufstellung zum eigenen Genus bis zur Varietät fiel! Ohne ein zu starres Festhalten an meiner Ansicht beurkunden zu wollen, glaube ich dennoch von meiner Meinung in den jetzigen Verhältnissen unserer scientiae amabilis so lange nicht abgehen zu müssen, bis wir uns nicht dahin ernstlich aussprechen, dass wir in dieser Hinsicht ein einheitliches Princip bei allen Gattungen mit unveränderlicher Consequenz durchführen wollen. Dann streiche ich die Segel und rufe nebst meinem herzlichsten Gruss Hrn. Juratzka zu: S i e haben vollk om men recht!

A g r a m, den 10. December 1857 .

\section{Correspondenzen.}

- 0 fen, im Jänner 1858. Zu Anfang des abgelaufenen Jahres erschien in den hiesigen sournalen folgender Artikel: "Unter den nambaften für wohlthätige und gemeinnützige $Z$ wecke vom Freiherrn $S$ i $m$ o n $S$ i n a gespendeten Summen befinden sich auch 4000 fl. C.M, welche der edle Spender dem k. k. Finanzrath Herrn La dis la u s v. Korizmics als Eigenthümer und Leiter des Journals: Gazdas ági la pok zur Verfügung angewiesen hat und zwar $2000 \mathrm{fl}$. zur Förderung nützlicher landwirthschaftlicher Forschungen und Erfindungen und $2000 \mathrm{fl}$. zu Prämien für die Lösung zeilgemässer landwirthschaftlicher Fragen. Um dem zweiten Theile dieser grossherzigen Widmung zu entsprechen, hat Herr v. Ko rizmics im Namen des Freiherrn von Sin a folgende Preisaufgaben ausgeschrieben: 1. Die Ausarbeitung einer geologischen Beschreibung Ungarns, 2. die Ausarbeitung eines auf die eigenen Studien und Erfahrungen des Verfassers gestützten botanischen Original-Werkes, 